\title{
The thermal expansion coefficient of monolayer, bilayer and trilayer graphene derived from the strain induced by cooling to cryogenic temperatures
}

Gregor A. McQuade, ${ }^{1,}$ a) Annette S. Plaut, ${ }^{1}$ Alan Usher, ${ }^{1}$ and Jens Martin ${ }^{2}$

1) Physics and Astronomy, University of Exeter, EX4 4QL, UK

${ }^{2)}$ Leibniz-Institut für Kristallzüchtung, 12489 Berlin, Germany

(Dated: 21 March 2021)

While thermally cycling monolayer, bilayer and trilayer graphene between $5 \mathrm{~K}$ and $300 \mathrm{~K}$ Raman spectroscopy has shown that cooling to $5 \mathrm{~K}$ induces a strain in these graphene flakes of $-0.081 \pm 0.003 \%$. This strain was used to measure the graphene thermal expansion coefficient (TEC) which was found to be $(-3.2 \pm 0.2) \times 10^{-6} \mathrm{~K}^{-1}$ for monolayers, $(-3.4 \pm 0.4) \times 10^{-6} \mathrm{~K}^{-1}$ for bilayers and $(-3.8 \pm 0.6) \times 10^{-6} \mathrm{~K}^{-1}$ for trilayers at room temperature. The TEC showed a similar temperature dependence across all graphene thicknesses and was found to be in good agreement with theoretical predictions. This study thus represents the first measurement of the TEC of bilayer and trilayer graphene. Modification of graphene flakes of all thicknesses with various electrical contact designs was found to have no significant impact on the resulting strain, and thus the TEC, compared to the pristine graphene.

Graphene represents the fundamental limit of twodimensional materials, consisting of single layers of carbon atoms arranged in a honeycomb bonding pattern. Since it was successfully isolated as a single layer in 2004, ${ }^{1}$ graphene has become of enormous interest for experimental study. Graphene devices have been used in various electromagnetic experiments involving low temperatures, exploiting the ballistic conduction behaviour of electrons in graphene. $\mathrm{Me}$ chanical strain has been shown to affect the electronic behaviour of graphene, and to generate pseudo-electromagnetic potentials $[$ It is therefore imperative to understand how temperature changes can strain graphene devices.

Strain in graphene devices can be thermally induced from the difference in thermal expansion between the graphene flake and its substrate. Graphene has been shown to have a negative thermal expansion coefficient (TEC),,-7$]$ and it is predicted to remain negative down to $0 \mathrm{~K}, \frac{8}{\text { giving rise to }}$ the possibility of the creation of significant strains over the temperature range between room and cryogenic temperatures. This expansion difference results in a biaxial strain which is produced due to the Van der Waals force exerted on the graphene by the surface of the comparatively thicker substrate with which the graphene is in contact (Fig. 11).

In this Letter, we report on the TEC's dependence on the temperature of monolayer, bilayer and trilayer graphene in the temperature range of 5-300 K, using the thermal strain of graphene on a supporting $\mathrm{Si} / \mathrm{SiO}_{2}$ substrate. This thus represents the first temperature measurement of the TEC of monolayer graphene below $30 \mathrm{~K}, \underline{6}$ and also represents the first measurement of the TEC of both bilayer and trilayer graphene accross any temperature range. Previous experimental and theoretical investigations into the TEC of graphene have produced a wide range of TEC temperature dependences, ranging from a positive TEC at all temperatures, 9 to a negative TEC up to temperatures of at least $1000 \mathrm{~K}, \frac{68}{6}$ with various disagreements over the negative to positive transition point. ${ }^{[47}$ This

\footnotetext{
a)Electronic mail: gm376@exeter.ac.uk
}

highlights the necessity for a definitive measurement of the graphene TEC, as an accurate measurement of the temperature dependence of the TEC of graphene is essential to create graphene devices with tailored thermal strain fields and hence pseudo-magnetic fields.

Raman spectroscopy is a versatile method for determining the properties of a material by probing its atomic lattice vibrations (phonons). In graphene, Raman spectra are regularly used to identify the number of graphene layers and to indicate the density of lattice defects. ${ }^{10}$ In this Letter, we use the strain-induced shifts in the phonon energies, which produce corresponding shifts in the G and 2D Raman peak energies, to measure the strain and TEC temperature dependences. The $G$ Raman peak is caused by the $E_{2 g}$ symmetry mode vibration of the graphene lattice, $11 / 12$ and the 2D Raman peak is the overtone of the $\mathrm{A}_{1 \mathrm{~g}}$ symmetry mode vibration, 13 the $\mathrm{D}$ Raman peak of the $A_{1 g}$ mode only being Raman active in the presence of lattice defects! 12

The graphene samples used in this study were exfoliated from highly ordered pyrolytic graphite (HOPG) onto silicon substrates with a $295 \mathrm{~nm} \mathrm{SiO}_{2}$ surface coating. Flakes with sides of length 6-20 $\mu \mathrm{m}$ were selected for fabrication and measurement. Graphene thicknesses were identified by the peak shape of the Raman 2D line which transitions from a single spectral peak for monolayer graphene to a combination of four sub-peaks in bilayer and trilayer graphene. ${ }^{12}$ Three styles of graphene devices were designed: isolated pristine graphene flakes supported by the substrate alone (Fig. 1p), two-point contacted graphene devices using gold (Fig. 11) and two-point contacted devices with gold "nails" through the graphene into the substrate (Fig. 1d). These designs were used to compare the different clamping effects that could be induced by standard electrical contacting.

Temperature-dependent Raman microscopy was undertaken in an optical cryostat with a temperature range of 4.2$300 \mathrm{~K}$. A long working distance objective with a $17 \mathrm{~mm}$ focal length was used to focus the laser beam onto the graphene flakes and collect the resulting Raman signal. Raman excitation was induced with a $514.5 \mathrm{~nm} \mathrm{Ar}^{+}$laser at an output 
power of $\leq 4 \mathrm{~mW}$ with a laser spot diameter of $6 \mu \mathrm{m}$ to avoid sample heating, which was confirmed by performing measurements at higher laser powers and observing no difference in the temperature-dependent energy shift behaviour of the Raman peaks.

The monolayer graphene $\mathrm{G}$ and 2D Raman peak energies were derived from fitted Lorentzian functions. These fits and the temperature dependence of the Raman peak energies of pristine monolayer graphene are shown in Fig. 2

In order to isolate the strain contributions to the Raman peak energy shift, the doping and intrinsic thermal effects on the Raman peak energy positions must each be accounted for and removed:

The intrinsic phonon thermal energy shift arises from the variation in the phonon self-energy with temperature ${ }^{14}$ This energy shift is not expected to vary between samples, so the theoretically predicted energy shift (Fig. 2p - blue line) can be subtracted from the data points. This predicted energy shift

(a)

decreasing temperature:
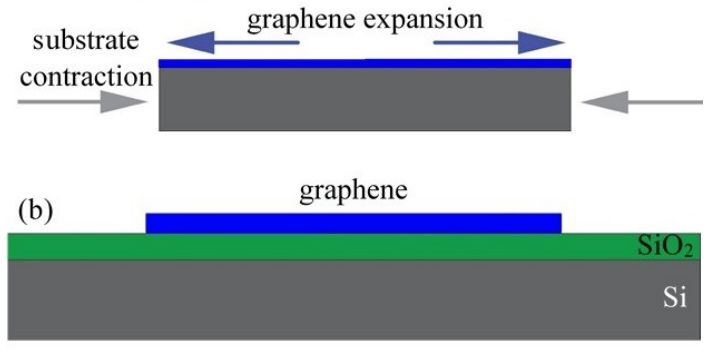

(c)

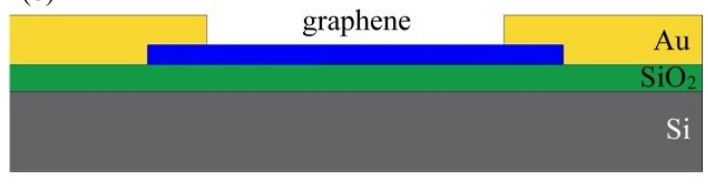

(d)

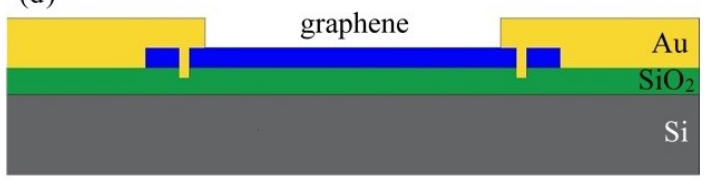

FIG. 1. Schematic representation of (a) thermal contraction, which results in compressive biaxial strain in the graphene as the temperature of the Si substrate (grey) and graphene (blue) is decreased; (b) pristine, (c) contacted and (d) "nailed" sample designs with the substrate oxide coating $\left(\mathrm{SiO}_{2}\right)$ shown in green, and the gold $(\mathrm{Au})$ contacts in yellow. has been approximated as a third-order polynomial! 15

$$
\Delta \omega_{\mathrm{G}}=-4.23 \times 10^{-4} T-3.03 \times 10^{-5} T^{2}+1.15 \times 10^{-8} T^{3},
$$

where $\Delta \omega_{\mathrm{G}}$ is the $\mathrm{G}$ Raman peak energy shift from the $0 \mathrm{~K}$ energy position, and $T$ is temperature. This has been used for the G Raman peak data, but not for the 2D Raman peak data as the magnitude of the intrinsic thermal 2D Raman peak energy shift is three times less than that of the G Raman peak over the temperature range investigated ${ }^{16}$ This results in the intrinsic thermal 2D Raman peak energy shift being comparable to the scatter in the data points in Fig. $2 \mathrm{~d}$ and therefore insignificant in comparison to the total temperature-dependent energy shift.

The $G$ to 2D Raman peak energy-shift ratio was then used to identify the strain-only energy shifts in the Raman peak energy positions separate from the doping contributions. Strain in graphene shifts the Raman peak energies by changing the bond lengths between the carbon atoms, causing an increase (decrease) in phonon energy with compressive (tensile) strain as determined by the Grüneisen parameter of graphene! $17 / 18$ The hydrostatic, biaxial-strain energy shift was measured independently of doping and temperature by Zabel et al. ${ }^{19}$ to be $57 \mathrm{~cm}^{-1} / \%$ for the $\mathrm{G}$ Raman peak and $140 \mathrm{~cm}^{-1} / \%$ for the $2 \mathrm{D}$ Raman peak. This results in a ratio of 2.45 between the strainonly energy shift of the 2D Raman peak to the G Raman peak as is shown by the red lines in Figs. 3 a-c.

Finally, changes in doping concentration also cause the $G$ and 2D Raman peaks to shift in energy. Shifts in the graphene Fermi energy away from the crossover point of the valence and conduction bands has been shown to increase the energy of the $\mathrm{G}$ and 2D Raman peaks. ${ }^{[2]}$ Doping of graphene on $\mathrm{Si} / \mathrm{SiO}_{2}$ substrates tends to be positive (hole) doping due to the oxide surface of the substrate ${ }^{21 \mid 22}$ For doping concentrations below $10^{13} \mathrm{~cm}^{-2}$, the Raman peak energy shift with doping concentration can be approximated as linear. ${ }^{20}$ A measure of Raman peak energy shift with doping by Das et al. ${ }^{20}$ shows that the 2D Raman peak shifts in energy with doping with a slope of 0.7 that of the G Raman peak. This gradient was used to selectively remove the doping contribution to the Raman peak energy positions. This doping-only energy-shift ratio is shown by blue lines in Fig. 3 h-c.

The use of these G to 2D Raman energy-shift ratios to separate the strain and doping contribution in monolayer graphene is demonstrated in Fig. 3a, and follows the approach of Lee $e t$ al. ${ }^{23}$ The zero energy shift position is assigned according to a Raman measurement on suspended graphene, which has minimal intrinsic strain and doping due to being isolated from external substrate effects: $1582 \mathrm{~cm}^{-1}$ for the $\mathrm{G}$ Raman peak and $2677 \mathrm{~cm}^{-1}$ for the 2D Raman peak ${ }^{23}$ Thus the doping contribution to the Raman peak energy shift with temperature shown in Fig. $2 k$ and $2 \mathrm{~d}$ can be removed by shifting each correlated data point along a vector parallel to the "doping only" $\left(n_{\mathrm{h}}\right)$ line until it lies upon the "strain only" $(\varepsilon)$ line, as shown in Fig. 3 . These adjusted Raman peak energy shift values are then translated into strain using the Grüneisen parameter ${ }^{[17 \mid 18}$ The biaxial Grüneisen parameters for graphene have not been definitively determined. Here, consistent with the literature, ${ }^{49 \mid 15}$ it has been assumed that the Grüneisen parameters do not vary with temperature and the value of the Grüneisen parameter of 

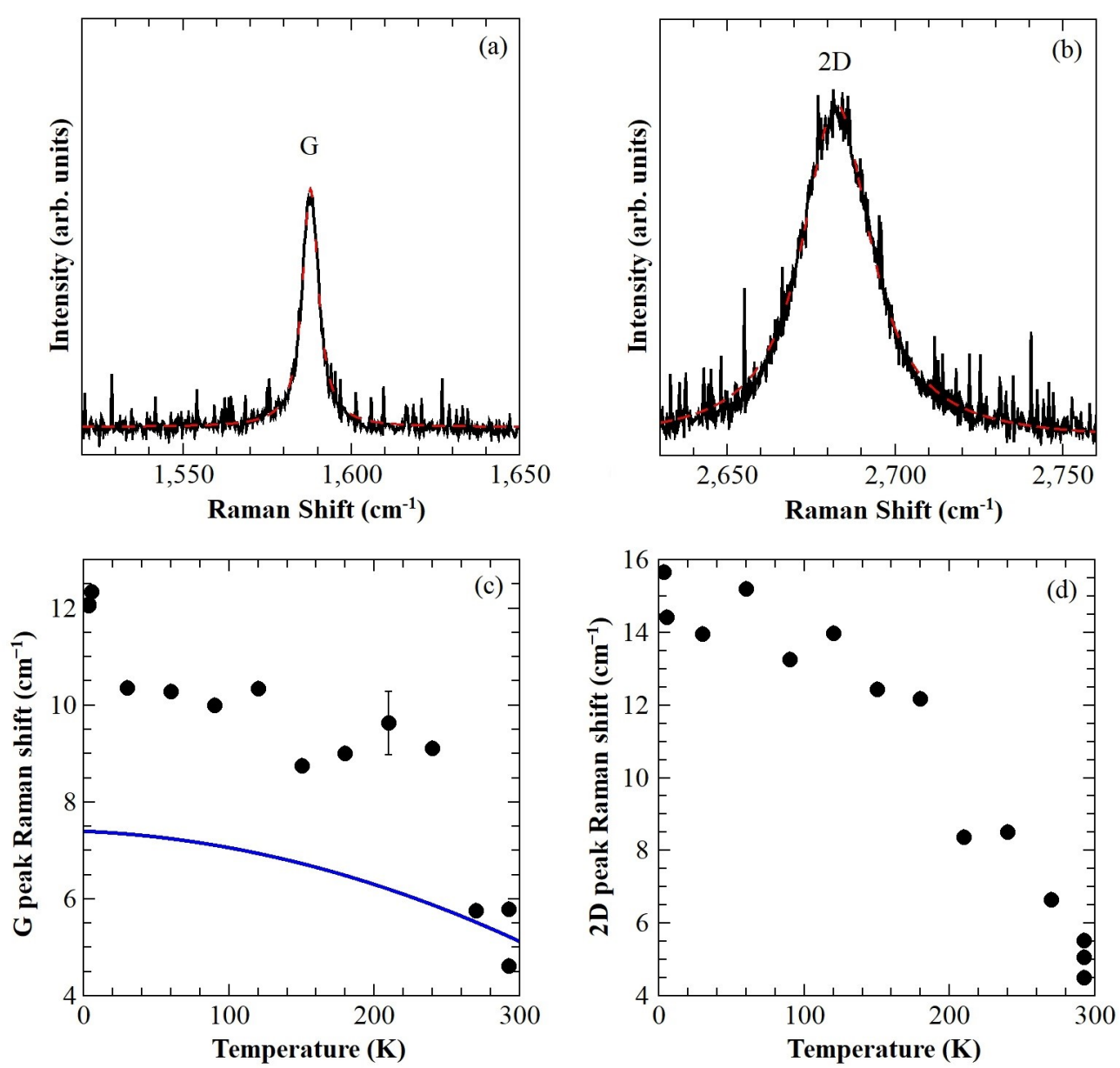

FIG. 2. (a) The G and (b) the 2D Raman peaks of pristine monolayer graphene (black solid lines) on the same intensity scale, with a Lorentzian curve fit to the data (red dashed lines). (c) Shift of the G Raman peak energy with temperature from the zero-strain and zero-doping Raman shift values. The predicted thermal phonon self-energy shift calculated by Bonini et al ${ }^{14}$ is shown as a blue line, offset to coincide with the data at $300 \mathrm{~K}$. (d) Shift of the 2D Raman peak energy with temperature from the zero-strain and zero-doping Raman shift values.

the G Raman peak $\gamma_{\mathrm{G}}$, has been taken to be 1.8 for biaxially strained monolayer graphene as derived from mechanical deformation experiments 19

This correlation process was repeated for bilayer and trilayer graphene although slightly modified: For bilayer graphene, a strain gradient of 2.4 and a doping gradient of 0.62 were used ${ }^{192425}$ The 2D Raman peaks for bilayer and trilayer graphene consist of multiple sub-peaks, therefore, for the purposes for these calculations, the 2D Raman peak energy position was taken to be the energy position of the envelope of the unresolved sub-peaks from fitting the envelope with a single Gaussian function. This is justified as it was found that the 2D Raman peak shape did not change with strain, $\frac{19}{19}$ i.e. that all its sub-peaks shift in energy in parallel with temperature. A zerostrain-doping Raman peak energy position of $1567 \mathrm{~cm}^{-1}$ and $2669 \mathrm{~cm}^{-1}$ from a suspended bilayer graphene flake measurement was used for the $\mathrm{G}$ and $2 \mathrm{D}$ Raman peaks respectively. 26 For trilayer graphene, a strain gradient of 2.5 and zero-straindoping Raman peak energy positions of $1567 \mathrm{~cm}^{-1}$ and 2621 $\mathrm{cm}^{-1}$, respectively, were used from another suspended flake measurement. ${ }^{24 \mid 27}$ The doping gradient of bilayer graphene was used for the trilayer graphene correlation as it is not expected to be significantly different within our experimental uncertainties. The value of $\gamma_{\mathrm{G}}$ for biaxially strained bilayer graphene has been taken to be 1.82 and $\gamma_{\mathrm{G}}=1.45$ for biaxially strained trilayer graphene, again derived from mechanical deformation experiments. ${ }^{24}$

The resultant strain dependence for pristine monolayer graphene is shown in Fig. $3 \mathrm{~d}$ and demonstrates a compressive strain as the temperature is decreased with a magnitude of $-0.081 \pm 0.003 \%$ at $0 \mathrm{~K}$, when averaged over 23 flakes, which is consistent with a negative TEC for graphene over the investigated temperature range. The samples showed no consistent variation in strain behaviour that correlated with the different device designs employed and depicted in Fig. 1p-d.

The TEC of graphene was calculated from the derivative of the strain with respect to temperature ${ }^{4}$

$$
\alpha_{\text {graphene }}=\alpha_{\mathrm{Si}}-\frac{\mathrm{d} \varepsilon}{\mathrm{d} T}
$$

where $\alpha$ is the TEC. The strain dependences were fitted with 

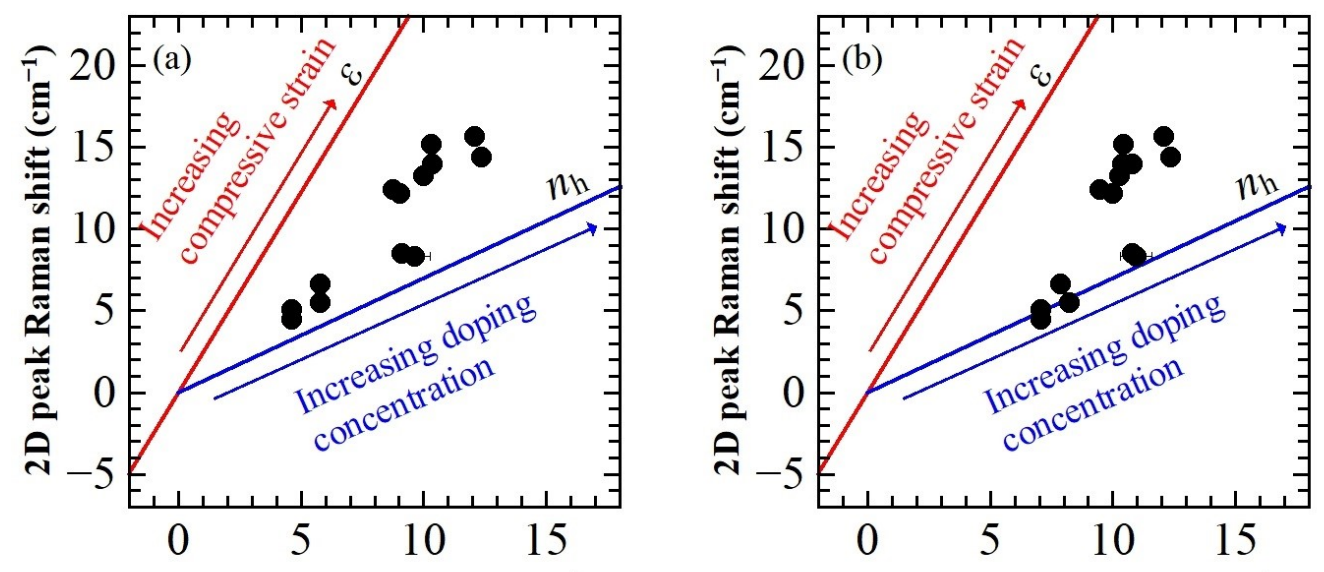

G peak Raman shift $\left(\mathrm{cm}^{-1}\right)$

G peak Raman shift $\left(\mathrm{cm}^{-1}\right)$
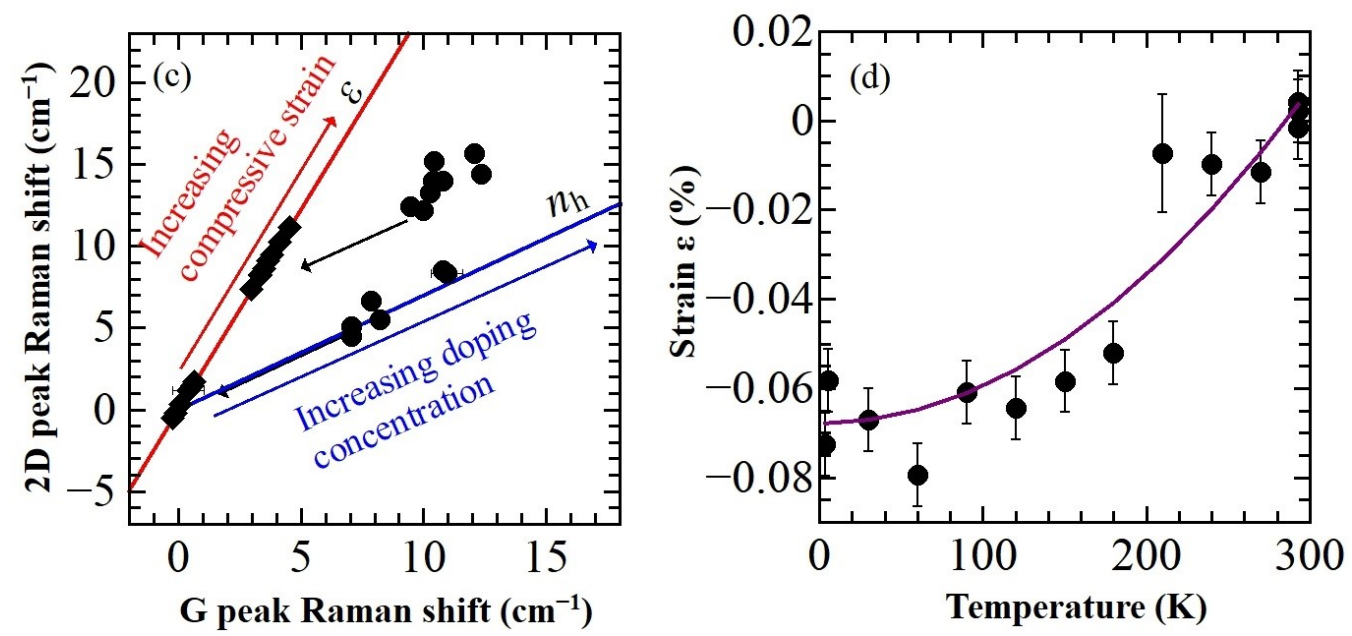

FIG. 3. (a) Correlation of the G and 2D Raman peak energies of Fig. 2k and 2 1 . (b) Shifted correlation of the data shown in (a) after removal of the intrinsic G Raman peak energy shift with temperature - see text. (c) Removal of the doping contribution to the correlation position by shifting the correlated data (circles) to the strain line (diamonds). (d) Isolated strain dependence of pristine monolayer graphene on temperature from (c). The data are then fitted to the quadratic equation: $\varepsilon=-0.0678+8.3 \times 10^{-7} T^{2}$ which is shown as a purple line.

a $T^{2}$-function (Fig. 3 $\mathrm{d}$ ) of the form

$$
\varepsilon=c+m T^{2}
$$

where $c$ is a constant and $m$ is the gradient. The lowest temperature data point was used to define the constant term $c$, leaving the gradient as the only fitting parameter. A higher order polynomial fit was not justifiable due to the error bars on the strain dependence data points in Fig. $3 \mathrm{~d}$. The quadratic form of Eqn. 3 results in a linear dependence of the derivative term in Eqn. 2. This in turn results in the slightly anomalous behaviour in the final calculated TEC dependence on temperature that mimics the features of the silicon TEC temperature dependence, $\alpha_{\mathrm{Si}}$ (Fig. 4 h). ${ }^{28}$

Fig. $4 \mathrm{a}$ shows the averaged TEC measurements across the one-, two- and three-layer graphene samples over the temperature range of 5-300 K. The figure shows good agreement with the theoretically calculated temperature dependence of the TEC by Mounet $e t$ al., 8 shown as a purple line, as well as a broad similarity across all three graphene thicknesses. This is in contrast to previous TEC measurements of monolayer graphene which have demonstrated a more negative
TEC dependence than predicted, 4,617 a rapidly varying,, 7 or even a positive TEC over the equivalent temperature range investigated ${ }^{[59}$ Our study also represents the first measurement of the bilayer and trilayer graphene TEC. At room temperature, the measured TECs were found to be $(-3.2 \pm 0.2) \times$ $10^{-6} \mathrm{~K}^{-1}$ for monolayers, $(-3.4 \pm 0.4) \times 10^{-6} \mathrm{~K}^{-1}$ for bilayers and $(-3.8 \pm 0.6) \times 10^{-6} \mathrm{~K}^{-1}$ for trilayers.

Additionally, it was demonstrated that the strain, and therefore the TEC, temperature dependence did not vary significantly either between the graphene device designs investigated in this study: A single monolayer graphene flake was separated into the three device designs of Fig. $1 \mathrm{p}-\mathrm{d}$ and the resultant TEC temperature dependences of these three devices are shown in Fig. 4b. There is an overlap in the uncertainty bounds of the TECs from these three different sample designs, which shows that these design differences are not the source of either the strain nor the TEC variation observed across the range of samples investigated in this study (which were averaged in Fig. (4a). It was therefore concluded that any sample variance was a result of the exfoliation process and hence the 

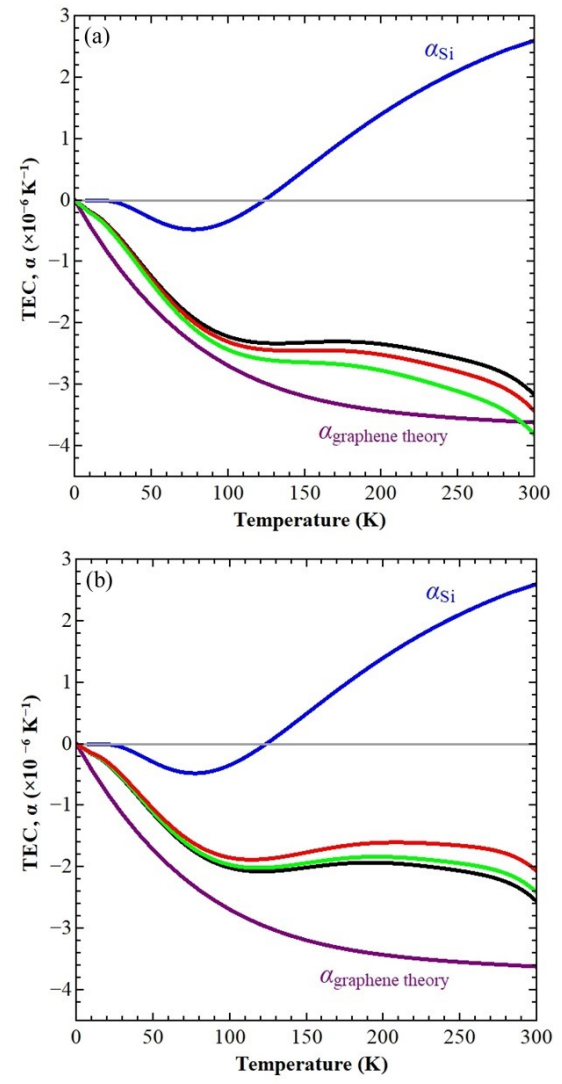

FIG. 4. (a) Averaged TECs ( $\alpha$ ) of monolayer graphene (black line), bilayer graphene (red line), and trilayer graphene (green line). (b) TECs $(\alpha)$ of a single monolayer graphene flake from which three differently contacted devices were made: pristine (black line), contacted (green line), and "nailed" (red line). The theoretical TEC of monolayer graphene is shown in purple, $\frac{8}{}$ and the TEC of silicon in blue for comparison. 28

degree that the graphene was in contact with the $\mathrm{Si} / \mathrm{SiO}_{2}$ substrate, rather than the actual device contact design, over the temperature range investigated $(5-300 \mathrm{~K})$.

While largely agreeing with the theoretical TEC,, 8 the TEC temperature dependences deduced in Fig. 4 all tend to be smaller in magnitude than the theoretical prediction. 8 This can be understood to be the case if the graphene flakes are not in perfect contact with the substrate surface, as depicted schematically in Fig. 5. In this scenario, the atomically rough surface of the $\mathrm{SiO}_{2}$ allows the graphene a certain amount of thermal expansion free from strain, resulting in a less negative TEC. The difference between the expected strain from the theoretical TEC and the measured strains was used to estimate the substrate surface roughness by modelling the strainfree relaxation of the graphene flake as the length difference between a chord and an arc on a circle, approximated as!

$$
s \approx \sqrt{d^{2}+\frac{16}{3} h^{2}}
$$

where $s$ and $d$ are the arc and chord lengths respectively, and $h$ is the height of the arc. Using chord length values

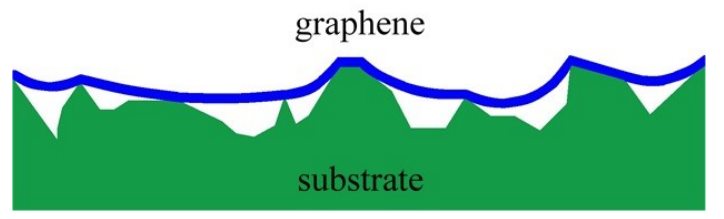

FIG. 5. Schematic representation of imperfect contact between graphene and $\mathrm{SiO}_{2}$ surface which allows the graphene sheet to expand without strain upon cooling - see text.

of $25-50 \mathrm{~nm}$ to represent the peak separation in the surface roughness of $\mathrm{SiO}_{2}$ surfaces as measured by atomic force microscopy (AFM), $\stackrel{30}{0}$ the arc height values obtained were consistent with a substrate surface roughness of $0.29-0.57 \mathrm{~nm}$. This roughness, calculated from our measured strain, is found to be consistent with AFM data. 30

In summary, the thermal strain in exfoliated graphene and its corresponding TEC was measured over the temperature range of 5-300 $\mathrm{K}$ from the shift in phonon energy measured by Raman spectroscopy for monolayer, bilayer and trilayer graphene and three device designs. The strain difference induced by cooling the monolayer graphene flakes on $\mathrm{Si} / \mathrm{SiO}_{2}$ substrates from room temperature to $5 \mathrm{~K}$ was found to be $-0.081 \pm 0.003 \%$ averaged over 23 flakes. The measured TECs: $(-3.2 \pm 0.2) \times 10^{-6} \mathrm{~K}^{-1}$ for monolayers, $(-3.4 \pm 0.4) \times 10^{-6} \mathrm{~K}^{-1}$ for bilayers and $(-3.8 \pm 0.6) \times 10^{-6} \mathrm{~K}^{-1}$ for trilayers at room temperature, were found to be in good agreement with the theoretical predictions of Mounet et al. ${ }^{[8}$ and consistent with a negative TEC across the whole investigated temperature range $(5-300 \mathrm{~K})$. However no significant difference was found to be induced by the style of electrical contacting due to the metal contacts clamping the graphene flake in any way. These results should inform further studies involving graphene devices that undergo temperature variations to create desired strain fields and hence pseudo-magnetic fields.

The data that support the findings of this study are available from the corresponding author upon reasonable request.

This work was funded by a Leverhulme Trust Research Project Grant.

${ }^{1}$ A. K. Geim and K. Novoselov, "The rise of graphene," Nat. Mater. 6, 183191 (2007)

${ }^{2}$ A. H. Castro Neto, F. Guinea, N. M. R. Peres, K. S. Novoselov, and A. K. Geim, "The electronic properties of graphene," Rev. Mod. Phys. 81, 109162 (2009) 
${ }^{3}$ F. Guinea, M. I. Katsnelson, and A. K. Geim, "Energy gaps and a zero-field quantum hall effect in graphene by strain engineering," Nat. Phys. 6, 30-33 (2010)

${ }^{4}$ D. Yoon, Y.-W. Son, and H. Cheong, "Negative thermal expansion coefficient of graphene measured by Raman spectroscopy," Nano Lett. 11, 3227-3231 (2011)

${ }^{5}$ I. R. Storch, R. De Alba, V. P. Adiga, T. S. Abhilash, R. A. Barton, H. G. Craighead, J. M. Parpia, and P. L. McEuen, "Young's modulus and thermal expansion of tensioned graphene membranes," Phys. Rev. B 98, 085408 (2018)

${ }^{6}$ V. Singh, S. Sengupta, H. S. Solanki, R. Dhall, A. Allain, S. Dhara, P. Pant, and M. M. Deshmukh, "Probing thermal expansion of graphene and modal dispersion at low-temperature using graphene nanoelectromechanical systems resonators," Nanotechnology 21, 165204 (2010)

${ }^{7}$ W. Bao, F. Miao, Z. Chen, H. Zhang, W. Jang, C. Dames, and C. N. Lau, "Controlled ripple texturing of suspended graphene and ultrathin graphite membranes," Nat. Nanotechnol. 4, 562-566 (2010)

${ }^{8}$ N. Mounet and N. Marzari, "First-principles determination of the structural, vibrational and thermodynamic properties of diamond, graphite and derivatives," Phys. Rev. B 71, 205214 (2005)

${ }^{9}$ S. Linas, Y. Magnin, B. Poinsot, O. Boisron, G. D. Förster, V. Martinez, R. Fulcrand, F. Tournus, V. Dupuis, F. Rabilloud, L. Bardotti, Z. Han, D. Kalita, V. Bouchiat, and F. Calvo, "Interplay between Raman shift and thermal expansion in graphene: Temperature-dependent measurements and analysis of substrate corrections," Phys. Rev. B 91, 075426 (2015)

${ }^{10}$ A. C. Ferrari and D. M. Basko, "Raman spectroscopy as a versatile tool for studying the properties of graphene," Nat. Nanotechnol. 8, 235-246 (2013)

${ }^{11}$ M. S. Dresselhaus, G. Dresselhaus, and A. Jorio, Group Theory: Application to the Physics of Condensed Matter (Springer, New York, 2008).

${ }^{12}$ A. C. Ferrari, J. C. Meyer, V. Scardaci, C. Casiraghi, M. Lazzeri, F. Mauri, S. Piscanec, D. Jiang, K. S. Novoselov, S. Roth, and A. K. Geim, "Raman spectrum of graphene and graphene layers," Phys. Rev. Lett. 97, 187401 (2006)

${ }^{13}$ A. C. Ferrari, "Raman spectroscopy of graphene and graphite: Disorder, electron-phonon coupling, doping and nonadiabatic effects," Solid State Commun. 143, 47-57 (2007)

${ }^{14}$ N. Bonini, M. Lazzeri, N. Marzari, and F. Mauri, "Phonon anharmonicities in graphite and graphene," Phys. Rev. Lett. 99, 176802 (2007)

${ }^{15}$ T. G. A. Verhagen, K. Drogowska, M. Kalbac, and J. Vejpravova, "Temperature-induced strain and doping in monolayer and bilayer isotopically labeled graphene," Phys. Rev. B 92, 125437 (2015)

${ }^{16}$ A. T. Apostolov, I. N. Apostolova, and J. M. Wesselinowa, "Temperature and layer number dependence of the $\mathrm{G}$ and $2 \mathrm{D}$ phonon energy and damping in graphene,"J. Phys.: Condens. Matter 24, 235401 (2012)
${ }^{17}$ T. M. G. Mohiuddin, A. Lombardo, R. R. Nair, A. Bonetti, G. Savini, R. Jalil, N. Bonini, D. M. Basko, C. Galiotis, N. Marzari, K. S. Novoselov, A. K. Geim, and A. C. Ferrari, "Uniaxial strain in graphene by Raman spectroscopy: G peak splitting, grüneisen parameters and sample orientation," Phys. Rev. B 79, 205433 (2009)

${ }^{18} \mathrm{G}$. Grimvall, Thermophysical Properties of Materials (North-Holland, Amsterdam, 1986).

${ }^{19}$ J. Zabel, R. R. Nair, A. Ott, T. Georgiou, A. K. Geim, K. S. Novoselov, and C. Casiraghi, "Raman spectroscopy of graphene and bilayer under biaxial strain: Bubbles and balloons," Nano Lett. 12, 617-621 (2012)

${ }^{20}$ A. Das, S. Pisana, B. Chakraborty, S. Piscanec, S. K. Saha, U. V. Waghmare, K. S. Novoselov, H. R. Krishnamurthy, A. K. Geim, A. C. Ferrari, and A. K. Sood, "Monitoring dopants by Raman scattering in an electrochemically top-gated graphene transistor," Nat. Nanotechnol. 3, 210-215 (2008)

${ }^{21}$ S. Pisana, M. Lazzeri, C. Casiraghi, K. S. Novoselov, A. K. Geim, A. C. Ferrari, and F. Mauri, "Breakdown of the adiabatic Born-Oppenheimer approximation in graphene," Nat. Mater. 6, 198-201 (2007)

${ }^{22}$ C. Casiraghi and S. Pisana, "Raman fingerprint of charged impurities in graphene," Appl. Phys. Lett. 91, 233108 (2007)

${ }^{23}$ J. E. Lee, G. Ahn, J. Shim, Y. S. Lee, and S. Ryu, "Optical separation of mechanical strain from charge doping in graphene," Nat. Commun. 3, 1024 (2012)

${ }^{24}$ C. Androulidakis, E. N. Koukaras, J. Parthenios, G. Kalosakas, K. Papagelis, and C. Galiotis, "Graphene flakes under controlled biaxial deformation,"Sci. Rep. 5, 15219 (2015)

${ }^{25}$ A. Das, B. Chakraborty, S. Piscanec, S. Pisana, A. K. Sood, and A. C. Ferrari, "Phonon renormalization in doped bilayer graphene," Phys Rev. B 79, 155417 (2009)

${ }^{26}$ C.-W. Huang, B.-J. Lin, S.-Y. Juang, F.-Y. Shih, W.-H. Wang, C.-Y. Liu, and H.-C. Chui, "Probing 2D sub-bands of bi-layer graphene," RSC Adv. 4, 51067-51071 (2014)

${ }^{2 /}$ C. H. Lui, Z. Ye, C. Keiser, E. B. Barros, and R. He, "Stacking-depended shear modes in trilayer graphene," Appl. Phys. Lett. 106, 041904 (2015)

${ }^{28}$ T. Middelmann, A. Walkov, G. Bartl, and R. Schödel, "Thermal expansion coefficient of single-crystal silicon from $7 \mathrm{k}$ to 293 K," Phys. Rev. B 92, 174113 (2015)

${ }^{29} \mathrm{~J}$. W. Harris and H. Stöcker, Handbook of Mathematics and Computational Science (Springer-Verlag, New York, 1998).

${ }^{30}$ V. Geringer, M. Liebmann, T. Echtermeyer, S. Runte, M. Schmidt, R. Rückamp, M. C. Lemme, and M. Morgenstern, "Intrinsic and extrinsic corrugation of monolayer graphene deopsited on $\mathrm{SiO}_{2}$, , Phys. Rev. Lett. 102, $076102(2009)$ 